\title{
The Mental and Physical Health Effects of Torture: The Role of Identity Salience as a Pathway to Posttraumatic Growth and Healing: The Case of Syrian Refugees and IDPs
}

\author{
Ibrahim Kira ${ }^{1,2^{*}}\left(\mathbb{D}\right.$, Hanaa Shuwiekh ${ }^{2,3}$, Boshra Al Ibrahim4, Jakoub Aljakoub ${ }^{5}$ \\ ${ }^{1}$ Center for Cumulative Trauma Studies, Stone Mountain, USA \\ ${ }^{2}$ The Center for Stress, Trauma and Resiliency, Georgia State University, Atlanta, USA \\ ${ }^{3}$ Fayoum University, Fayoum, Egypt \\ ${ }^{4}$ The Kuwait Ministry of Health (MOH), Kuwait, Kuwait \\ ${ }^{5}$ Sabah Hospital, Kuwait, Kuwait \\ Email: ^kiraaref@aol.com, hanaashiwk2000@hotmail.com, bushora20855@gmail.com,dr.jakoub7979@gmail.com
}

How to cite this paper: Kira, I., Shuwiekh, H., Al Ibrahim, B., \& Aljakoub, J. (2021). The Mental and Physical Health Effects of Torture: The Role of Identity Salience as a Pathway to Posttraumatic Growth and Healing: The Case of Syrian Refugees and IDPs. Psychology, 12, 1825-1847.

https://doi.org/10.4236/psych.2021.1211110

Received: October 3, 2021

Accepted: November 26, 2021

Published: November 29, 2021

Copyright $\odot 2021$ by author(s) and Scientific Research Publishing Inc. This work is licensed under the Creative Commons Attribution International License (CC BY 4.0).

http://creativecommons.org/licenses/by/4.0/

\begin{abstract}
Objective: Some studies found that torture did not predict posttraumatic stress disorder (PTSD) and is associated with more complex symptoms and that torture survivors are more resilient than other refugees. The goal is to recheck the validity of these findings. Method: A secondary analysis of data of 502 Syrian refugees, of whom 123 were torture survivors, we measured cumulative stressors and traumas (CST), PTSD, identity salience, post-cumulative trauma-related disorders (P-CTD), posttraumatic growth (PTG), and physical health. We conducted independent samples t-test between tortured and non-tortured refugees and hierarchical multiple regression analyses with PTSD, P-CTD, and PTG as dependent variables. We entered intersected discrimination, torture, and identity salience in the last step. Results: Independent samples t-test indicated that torture survivors have more neurological and musculoskeletal problems, higher psychoticism-dissociation, suicidality, and executive control deficits. There were no differences between the two groups in PTG. Further, torture survivors have higher identity salience than other nontortured refugees. Regression analysis indicated that torture and intersected discrimination were predictors of P-CTD syndromes; torture was a stronger predictor. Torture did not predict PTSD. Identity salience was a strong predictor of PTG (Beta $=.51)$. Conclusions: Torture survivors may develop PTSD because of other traumas other than torture. The results clarified some of the mechanisms that linked high identity salience and PTG in torture survivors, even though they have worse physical health, higher trauma load, and
\end{abstract}


mental health symptom severity, compared to other refugees. Torture survivors' stronger collective identity empowered their PTG. The clinical implications of the results were discussed.

\section{Keywords}

Torture Survivors, Refugees, Identity Salience, PTSD, Dissociation, Executive Function Deficits

The Study Significance Statement: The study advances our understanding of the impact of torture and other traumatic experiences on torture survivors and the contribution of their strong group identity and its salience to posttraumatic growth. While torture survivors have higher trauma and physical and mental symptoms' load than other non-tortured refugees, they have a higher or equal level of posttraumatic growth and stronger group identity commitment that helped them with their PTG. The results guide clinicians to refocus on the identity factor in treating torture survivors.

\section{Introduction}

Torture is not a single traumatic event; it is a complex cumulative traumatic experience that may include various types and traumatic dynamics (e.g., Kira et al., 2013c; Silove, 1999). Torture has physical and psychological consequences. Physically, it can severely affect neurological and musculoskeletal systems (e.g., Moreno \& Grodin, 2002; Olsen et al., 2006). Psychologically, unlike other personal and interpersonal traumas, torture is a collective (or group) identity trauma that can trigger different existential anxieties and activate the salience of the tortured person's group identity. A torture survivor has been tortured because he/she belongs to a group, and the salience of his/her group identity is an essential factor. Governments and political groups practice torture for various purposes, such as extracting information (e.g., Shue, 1978) or punishing, intimidating, silencing, or coercing social minorities or political opposition groups and their members (e.g., Summerfield, 2003). However, various definitions of torture include exacting physical and mental pain on an individual representing a group by others who have an official role or physical or temporal charge/control. Kira (2017) had recently redefined torture to include, in addition to acts by government agents, the acts of non-state actors such as rebels. According to this definition, torture is "an act of inflicting physical or and mental suffering on a person by other/s who has/have a legal capacity or physical or temporal control, in the context of explicit or implicit inter- or intragroup discord or conflict other than in the case of lawful nonpolitical sanctions)" (Kira, 2017: p. 328). Studies identified the torture experience structure to include psychological torture, sexual torture, harsh and abusive interrogations, physical torture, and extreme sensory stimulation (Hooberman et al., 2007; Kira, 2017). 
Torture prevalence among refugee and asylum seekers, including Syrian refugees, is estimated above $30 \%$ (Kalt et al., 2013). Prior research on refugees and torture survivors has suggested that the mental health's most reliable predictors for those who sought refuge in other countries and did not continue to live in the countries of their torture include pre-refuge, refuge, and post-refuge stressors and traumas. Some early studies found that pre-refuge adversities accounted for most of the variance in mental health symptoms (e.g., Nicholson, 1997; Steel et al., 1999). Conversely, the more recent research determined that the post-refuge stressors, especially the ongoing intersected discrimination in the host countries, to be more salient, accounting for more variance (Chu et al., 2013; Edge \& Newbold, 2013; Kim et al., 2019; Kira et al., 2010). Intersected discriminations are various micro and macro-aggressions types that target simultaneously different aspects of the person's identity, for example, race, color, religion, gender, sexual preference, national origin, poverty, or status, or belonging to opposition or minority group (e.g., Kira et al., Paper Submitted for Publication). The cumulative impact of pre-refuge, refuge, post-refuge traumas have cumulative dynamics that negatively affect torture survivors' physical and mental health (e.g., Abu Suhaiban et al., 2019; Choi et al., 2017; Kira et al., 2013c; Song et al., 2018; Steel et al., 2009). The stressors and traumas incremental accumulation process may entail, as in the case of torture survivors and refugees, different dynamics than the current focus on single traumas prescribed in Criterion "A" of PTSD and yield more complex symptoms such as complex PTSD (C-PTSD) and post cumulative trauma disorders (P-CTD) or (CTD).

C-PTSD refers to the constellation of symptoms that may result from prolonged, chronic exposure to traumatic experiences, especially in childhood, instead of PTSD, which is more typically associated with a discrete traumatic incident or set of traumatic events. C-PTSD consists of the PTSD symptom clusters (re-experiencing, avoidance and arousal) and self-organization disturbances (World Health Organization, ICD-11, 2018). While C-PTSD was proposed as a new diagnostic category, post-cumulative trauma disorders (P-CTD) or (CTD) was proposed as a comorbid cluster of different disorders that result from the cumulative effects of intersected identity and non-identity traumas (Kira et al., 2012b). P-CTD in torture survivors and refugees may include four clusters of comorbid syndromes: depression/anxiety/somatization comorbidity cluster, psychosis, and dissociation cluster, self-control and executive deficits cluster, and suicidality cluster (Kira, 2010; Kira et al., 2012b).

Life-time cumulative stressors and traumas in highly traumatized individuals and groups such as torture survivors and refugees rarely yield a single diagnosis but rather a profile of comorbid syndromes. The concept of complex PTSD was early suggested and recently codified in CD-11 may not be enough to describe the actual mental health impact of cumulative stressors in torture survivors and refugees. Post-cumulative trauma disorders (P-CTD) syndrome proposed earlier in the literature describes the cumulative impact of stressors and trauma in the lifetime in highly traumatized individuals and groups (Kira et al., 2012b). 
One refugee group that suffered severely is Syrian refugees. After the beginning of the Arab Spring and the Syrian civil war, many Syrian citizens have fled to neighboring countries due to violence and difficult living conditions, such as Turkey, Jordan, Lebanon, and Iraq. In the Syrian civil war, $60 \%$ of the country's prewar population was forced out of their homes in the worse refugee crises in recorded history, and hundreds of thousands were killed (e.g., Connor \& Krogstadl, 2016). The enormous adversities encountered by Syrians presented existential threats to their personal, physical, and collective identities (e.g., national) (e.g., Al-Ibraheem et al., 2017; Kira et al., 2017; Kira et al., 2019b).

The torture experience was variably associated with PTSD and depression (e.g., Johnson \& Thompson, 2008; Steel et al., 2009). However, studies by Kira et al., 2006, replicated by Hollifield et al., 2011, found that torture trauma did not predict PTSD after controlling previous traumas and ongoing intersected discriminations. Nevertheless, in further analysis, Kira, Ashby, et al. (2013c) found that torture and PTSD are associated non-linearly. That means that moderate torture stressors may predict PTSD, while severe torture stressors predict other more severe symptoms. Torture trauma is usually too distressing that tortured individuals may tend to block or overly suppress re-experiencing, which is a hallmark of PTSD (e.g., Varvin \& Rosenbaum, 2003). Such extreme suppression may yield more complicated syndromes than PTSD, such as severe dissociation, psychotic symptoms, executive function deficits, and suicidality (Kira et al., 2013c; Rathke et al., 2020). A longitudinal study found that ex-prisoners of war have persistent dissociation (Zerach et al., 2014). Rathke et al. (2020) found that about $30 \%$ of treatment-seeking refugees with PTSD have secondary psychotic symptoms. Torture survivors may have PTSD due to exposure to traumas other than torture or in addition to torture.

There are replicated empirical evidence of the nonlinear relationships between PTSD, trauma, and posttraumatic growth (Helgeson et al., 2006; Kanaan et al., 2019; Kira et al., 2013c; Kleim \& Ehlers, 2009). That may mean that those experiencing moderate levels of trauma and those experiencing moderate levels of PTSD have the highest level of growth, whereas those experiencing low and high levels of trauma or PTSD (e.g., torture survivors) have the lowest growth level. Conversely, a study found that torture survivors, due to their high group identity salience resulted from being tortured for their group of belonging, have more resilience, and higher social support than other non-tortured refugees (Kira et al., 2006).

One of the relatively poorly understood variables in most torture survivors and refugees' studies is the role of identity in mental health and PTG. Torture not only affects one's identity, but torture is also inflicted on the person because he/she belongs to a minority or opposition group. A group with identities that the dominant group negates is at increased risk of exposure to torture and adverse mental health conditions. Identity, a nonlinear dynamic system, is the center of personal agency, self-executive control, and executive functions (Elmore \& Oyserman, 2012; Kira, 2020; Kira, 2021a). Identity can be viewed as a lens through which individuals appraise and construct the events' meaning and 
then execute a response accordingly (Kira, 2019; Kira et al., 2019a; Kira et al., 2019b). Identity salience is the probability that a given identity will be invoked in social interaction (Stryker 1968). There is evidence of a strong link between strong ethnic identity and better mental health and PTG (e.g., Kira et al., 2021b; Kira \& Shuwiekh, 2021; Mossakowski, 2003). Commitment to different identities in different age groups was associated with better mental health in various previous studies (e.g., for meta-analysis, see Smith \& Silva, 2011). Identity and its dynamics are central to torture survivors' mental health and posttraumatic growth (PTG). There are some lingering questions about the dynamics of identity in the context of torture, intersected discrimination, and cumulative adversities. What are the variables that mediate the potential effects of identity salience on PTG? Are these dynamics invariant across genders? One study (Kira \& Shuwiekh, 2021) found that increased identity salience was significantly related to higher PTG, lower P-CTD, and PTSD.

There is empirical evidence the violation of identity (interested discrimination underlie the disproportionate impact of COVID-19 on minorities (Kira et al., 2021b). Our study aims to replicate and expand on previous results about the effects of torture on mental health and that torture survivors have higher PTG compared to other non-tortured refugees on a different sample of torture survivors. Further, the objective is to explore the mechanisms that empower torture survivors to develop higher or comparable PTG than non-tortured refugees, though they have higher trauma load and symptom severity than other refugees.

\section{Research Hypotheses}

Hypothesis 1: Torture survivors have higher health problems, especially in Neurological and Musculoskeletal systems, higher trauma load, and higher PTSD and P-CTD symptoms, compared to non-tortured, replicating previous results.

Hypothesis 2: Because Torture survivors have been tortured for their group of belonging, they have higher identity salience (group identity) than the non-tortured group who did not suffer as much for their group. They have higher or comparable posttraumatic growth regardless of their higher level of trauma load and mental and physical health symptom severity compared to other refugees who were not exposed to torture.

Hypothesis 3: Torture will not predict PTSD. It will not be associated with re-experiencing but with its complex symptoms of arousal and dissociation and will predict the complex post cumulative trauma disorder that includes the potentially more severe symptoms of psychoticism/dissociation, executive dyscontrol, and suicidality. That will replicate previous results.

Hypothesis 4: The intersected discriminations in refugees and torture survivors will predict PTSD.

Hypothesis 5: Torture survivors will have higher group identity salience than other refugees. Identity Salience will predict posttraumatic growth (PTG) in torture survivors, explaining their higher or comparable PTG compared to other 
less traumatized and less affected refugees who did not experience torture due to their belonging to the targeted group.

\section{Method}

Participants and procedures: For participants' gender, $32.3 \%$ were females. For marital status, $65.9 \%$ were married, $22.3 \%$ were single, $2.8 \%$ were divorced, $7.6 \%$ were widowers, and $1.4 \%$ were others. Age ranged from 17 to $78(\mathrm{M}=$ 35.76, SD = 10.93). For religion, $96 \%$ were Muslims, and 4\% were Christians. For education, $12.8 \%$ had minimum reading and writing skills, $8 \%$ were at elementary level education, $15.1 \%$ had intermediate level education, $18.9 \%$ had high school level education, $34.9 \%$ had a college or university degree, and $10.4 \%$ had a post-graduate degree. Power analyses $\left(G^{\star}\right.$ Powerprogram, version 3.1$)$ showed that the sample size was adequate for valid results (power $>.80, \mathrm{p}=.05$, effect size: $\mathrm{f} 2=.15$, see e.g., Mayr, Erdfelder, Buchner, \& Faul, 2007). The current study is a secondary analysis for data collected for different other studies (Al-Ibraheem et al., 2017; Kira et al., 2017; Kira et al., 2019b). The data was collected in February through April 1996, from displaced Syrians in Syria proper $(\mathrm{N}=195)$, and March through August 1996 from Syrian refugees in Nederland $(\mathrm{N}=111)$, and from June through August 1996 from Syrian refugees in Egypt $(\mathrm{N}=196)$. The number of torture survivors in this combined data set is $(\mathrm{N}=123)$, and the total number of the combined data set is 502. Trained graduate students in clinical psychology collected the data under direct supervision from advisors and the research team. Snowbowl sampling was used to collect the data individually and through organizations that serve refugees, such as mosques and churches (for details of each data set procedure, see Al-Ibraheem et al., 2017; Kira et al., 2017; Kira et al., 2019b).

Participation was voluntary; each person took approximately $25 \mathrm{~min}$ to complete the entire questionnaire, not including the clinical interview that used CAPS and P-CTD. Trained graduate students in clinical psychology administered CAPS. For details of the procedures, see Kira et al., 2019b. The IRB of the sponsoring university approved the research.

Previous studies that used these two combined data sets focused on exploring the effect of the Syrian conflict on Syrian refugees in the Nederland and IDPs mental and physical health (Al-Ibraheem et al., 2017) and investigating the impact of this conflict on the Syrian refugees in Egypt threatened national identity (Kira et al., 2017). Another study that used this combined data set examined the role of appraisal and emotion regulation in mediating the impact of cumulative stressors on PTG and mental health (Kira et al., 2019b). These three studies did not target torture survivors or measure the effects of torture on mental health or PTG. Then little or no overlap between the current study focus and the previous studies that utilized these data sets.

\section{Measures}

Syrian Oppressive Experience (SOE) Scale (Al Ibraheem et al., 2017). $t$ is a 
32-item scale that was developed specifically for the studies on Syrian refugees. It included three main types of events that represent the Syrian refugees' overwhelming stressors and traumatic experiences: torture stressors that include different types of torture, e.g., electric shock, forced position (13-items). The second primary stressors type included sustaining injuries during the violence and devoting time to go undercover to evade being captured. The third primary type of stressors was the secondary trauma resulting from witnessing brutal killings or having one or more family members injured or died in the atrocities. The scale represents all the stressors and traumatic stressors that Syrian refugees endured. The scale has a of 93 .

The Cumulative Stressors and Traumas Scale CST-S (short form) (Kira, et al., 2008). The CST-S is based on the development-based trauma framework (DBTF) (e.g., Kira, 2001; Kira, 2019; Kira, 2021a; Kira, 2021b; Kira et al., 2018; Kira et al., 2019c; Kira et al., 2013b). The scale (34 items version) is designed to measure seven types of stressors and traumas. Additionally, it includes three items that measure chronic and significant life stressors. The seven types of stressors/traumas include collective identity traumas (e.g., discrimination due to race, color, religion, sexual preference and cultural origin, oppression, and torture). They include personal identity trauma (e.g., early childhood traumas such as child neglect and abuse). They include status identity/achievement trauma (e.g., failed business, fired, and drop out of school) (non-criterion A traumas). They also include survival trauma (e.g., combat, car accidents, and natural disasters). They include attachment trauma (e.g., abandonment by parents), secondary trauma (i.e., indirect trauma impact on others), and gender discrimination. Added to these seven stressor and trauma types are intersected discrimination, which is the cumulative score of different discrimination types, including gender discrimination. An example of items of gender discrimination is "I was put down, denied my rights, or discriminated against in the society (not by family members), by some others' negative attitudes, stereotypes or actions, or by institutions because of my gender (being a girl/woman or a boy/man)". The CST-S evaluates cumulative stressors and traumas concerning its mere occurrence, frequency, type, and negative and positive appraisals, and age of happening. However, in this short survey study, we used only frequency and occurrence questions. For each question, participants are asked to specify their encounter of a stressor's (i.e., occurrence) (yes $=1$, no $=0$ ) and the frequency of happening on a 5 -point scale $(0=$ never; $4=$ many times). If participants indicated that they had experienced the stressor, they were asked to indicate the stressor's impact on a 7 -point scale $(1=$ extremely positive; $7=$ extremely negative). The CST-S includes two overall measures for cumulative stressors and traumas' dose: occurrence and frequency. Investigators can compute subscales for each of the stressor/trauma types. The CST-S has shown adequate internal consistency $(\alpha=.85)$, and test-retest stability (.95 in 4 weeks), and predictive, convergent, and divergent validity in different studies (e.g., Kira et al., 2019c; Kira et al., 2020a; Kira et al., 2013b; Kira et al., 2021a; Kira et al., 2018; Kira, 2021b). The measure has 
been translated and validated into different languages, including Arabic, Polish, Spanish, Turkish, Korean, Burmese, and Yoruba. In the present analysis, we used the cumulative stressors and trauma occurrence and intersected discrimination subscales. The cumulative stressors and traumas occurrence scale alpha is .86, and .70 for intersected discriminations subscale.

Identity Salience Scale (ISS). The ISS (Kira et al., 2011) is a 10-item scale developed in research with 880 Palestinian adolescents. The measure was used on adult Syrian refugees (e.g., Kira et al., 2019b). Identity dormancy/salience describes the person's specific identity (personal or group) status within the nested identity hierarchy, whether peripheral or central. It consists of questions like "I feel threatened personally by hate crimes committed against members of my race, religion, ethnic or cultural group or another group of my belonging or myself". Another question is, "Sometimes I wish to kill myself or somebody before my ethnic or religious group, or nation or any other group of my belonging eliminated, subjugated, or harmed". The response means how much they disagree or agree on a scale from 1 to $7(1=$ entirely disagree; $7=$ absolutely agree $)$. Higher scores mean higher collective identity salience; lower scores higher personal identity salience. Confirmatory and exploratory factor analysis confirmed two subscales: identity commitment and identity militancy. Internal consistency ( $\alpha$ ) for the measure was .80 for adolescents with $\alpha=.74$ for the Commitment and .75 for the Militancy (ready to die for your group) subscales. Test-retest reliability after three weeks was .76 . The scale's $\alpha=.88$ for the current sample ( .87 for Commitment and .82 for Militancy).

The Clinician-Administered PTSD Scale (CAPS-2) is a clinical interview that evaluates 17 symptoms rated on severity and frequency on a 5-point scale. The CAPS had established and robust psychometrics (Weathers et al., 2001). The instrument has four-factor subscales: avoidance, re-experiencing, arousal, and emotional numbness/dissociation. Betemps et al. (2003) found that the frequency dimension is associated with higher reliability and item discrimination than the intensity dimension. The current study used the frequency part of the scale, which most psychiatric research regularly uses. An interview format was used to administer the CAPS-2. In the current study, the measure has $\alpha=.91$.

Post-Cumulative Trauma-related Disorders Measure ( $P$-CTD). The P-CTD (Kira et al., 2012b) is a 16-item scale was created through research on five clinical and community studies of adolescent and adults refugees, African and Arab Americans. The CTD is an index scale containing comorbidity of 13 diverse syndromes, including loss of self-control, memory deficits, dissociation, somatization, visual and auditory hallucinations, paranoid ideation, and difficulties in concentration, and self-injury, suicidal ideation, plans or attempts to commit suicide. The participant marks each question on a scale from 0 (the behavior is not applicable); to 4 (the behavior is very much present). The confirmatory and exploratory factor analyses result confirmed four factors/constructs: executive control and self-control deficits factor, suicidality and self-harm construct, psy- 
choticism and dissociation factor, and depression/anxiety/somatization factor. The scale proved to have adequate internal consistency ( $\alpha=.85-.98)$. Test-retest consistency in a 6-week interval was .86. In the current study, $\alpha=.86$, and the for subscales, depression/anxiety/somatization comorbidity subscale, $\alpha=.70$; for psychoticism/dissociation subscale, $\alpha=.80$, for executive function-control subscale $\alpha=.74$, and suicidality subscale $\alpha=.77$.

The Post-Traumatic Growth Inventory (PTGI); (Tedeschi \& Calhoun, 1996) evaluate perceived positive life changes (e.g., enhanced relationships, greater life appreciation) following stressful experiences. In completing the 21 items, participants answer on a range from 0 (I did not experience the change as a result of my experience) to 5 (I experienced this change to a very significant degree). The inventory includes five subscales intended to assess: personal strength, relating to others, new possibilities, spiritual change, and appreciation of life. Tedeschi and Calhoun (1996) found that the total scale has an alpha of .90 and the test-retest consistency of .71. The inventory was found to have robust psychometrics in its Arabic version (Kira et al., 2012c, 2013a), with $\alpha=.96$. The inventory has $\alpha=.90$ in the current study.

Physical Health Scale (15 items) (modified) (Kira et al., 2001). The scale was initially created in studies on Palestinians and Iraqi refugees. The elevated score on the scale was associated with PTSD, CTD (complex PTSD), and older age (Kira et al., 2006). The lower the score, the better is the participant's health condition and vice versa. Various studies found that the scale had adequate reliability that ranged between 70 - 85. It consists of a question about self-rated health and a checklist of specific acute and chronic physical health problems, based on the taxonomy of health problems of ICD-9-CM codes for selected general medical conditions. Medical conditions include neurological and blood pressure disorders and digestive system, musculoskeletal, and endocrine illnesses. It included three questions on how health problems affected the participant's relations, memory, and ability to work. We will use the scale items to assess the differences between tortured and non-tortured individuals in different health conditions. The scale has $\alpha=.75$ in the current study.

Demographics: Gender, Age, education, marital status, religion, and income data were collected. Yearly income was converted to a score from $1-5$, with $1=$ $0-500 \$$, and $5=$ more than $5000 \$$ a year.

Data Analysis. We utilized IBM-SPSS 22 and Amos 22 software to analyze the data. Examination of the data showed that missing values were between .2 and $2.5 \%$, percentages below the $5 \%$ cut-off recommended by Tabachnick and Fidell (2013). We replaced missing values by means. We performed zero-order correlations between the main variables to examine if the correlations reflect our hypotheses for the association between torture and intersected discrimination and mental health variables. We performed independent samples $t$-test between tortured $(\mathrm{N}=123)$ and non-tortured groups $(\mathrm{N}=379)$. We used Cohen's formula to calculate Cohen's d effect size. Cohen (1992) suggested that effect sizes of .20 are small, .50 is medium, and .80 is large. The goal was to 
check our hypotheses on the differences between tortured and non-tortured groups in health, trauma load, mental health (PTSD and P-CTD), PTG, and identity salience.

To examine the effects of torture and intersected discrimination on mental health and the effects of identity salience on PTG, we conducted hierarchical multiple regression analyses. The data were assessed to ensure that the multivariate tests' assumptions were fulfilled (multicollinearity, homogeneity of covariance-variance matrices, and homogeneity of variances and linearity). We tested for collinearity, and the variance inflation factor (VIF) was less than 5.00 for all variables, suggesting no multicollinearity (e.g., Hair et al., 2017). PCTD and PTSD were the dependent variables in the analyses. Marital status, gender, age, education, and income were entered as independent variables in the first step. In the second step, we added Syrian civil war traumas. In the third step, we added intersected discrimination and torture. In another hierarchical multiple regression analysis, with PTG as a dependent variable, in the second step, we added identity salience as a predictor of PTG.

To convincingly test our hypotheses, we conducted multiple analyses (correlations, $t$-tests being, hierarchical regression). Such many analyses may increase type I errors which can affect the reliability of the findings. Because of this fact, we will only consider the significant results on the level of .01 or higher to avoid the potential increase in type 1 error (a false positive).

\section{Results}

Descriptive results: All torture survivors (100\%) endured at least three types of traumas $(M=10.88, S D=5.18)$; for non-tortured, $95.3 \%$ encountered at least one type of trauma $(\mathrm{M}=6.75, \mathrm{SD}=4.38)$. We utilized the cut-off criteria of a score $\geq 34$ of the PTSD scale to calculate probable PTSD; $37 \%$ of the torture sample and $26 \%$ for the non-tortured sample scored at or above the cut-off score. Further, $58 \%$ of the tortured sample scored at or above the cut point score of $\geq 22$, of Post-Cumulative Trauma Disorders (PCTD), compared to $36.5 \%$ for the non-tortured.

Independent samples t-test results between tortured and non-tortured: The tortured suffer significantly more physical health problems compared to nontortured. Torture survivors reported that their health conditions affected their relationships, the ability to work, and memory. They have significantly more neurological and musculoskeletal problems than non-tortured, consistent with the torture experience. Further, they have more blood pressure and heart problems, respiratory system, diabetes, and endocrinology problems than the non-tortured. There is no significant difference between them and non-tortured in digestive, reproductive, or other health problems (Table 1).

Further, they tend to appraise events more negatively. They have a much higher trauma load. They have higher PTSD and higher post-cumulative trauma disorders (PCTD). For the PTSD symptoms, there are no differences between 
Table 1. Independent samples t-test between the tortured and non-tortured in physical health conditions.

\begin{tabular}{|c|c|c|c|c|c|c|c|c|c|}
\hline Variable & Groups & $\mathrm{N}$ & Mean & $\mathrm{SD}$ & SE & $\begin{array}{c}\text { Mean } \\
\text { difference }\end{array}$ & $t$ & $p$ & $\begin{array}{l}\text { Cohen's d } \\
\text { effect size }\end{array}$ \\
\hline \multirow{2}{*}{$\begin{array}{l}\text { The effects of health on } \\
\text { personal relations }\end{array}$} & Non-tortured & 379 & 379 & 1.47 & 1.198 & \multirow{2}{*}{-.368} & \multirow{2}{*}{-2.88} & \multirow{2}{*}{.004} & \multirow{2}{*}{.29} \\
\hline & Torture Survivors & 123 & 123 & 1.84 & 1.321 & & & & \\
\hline \multirow{2}{*}{$\begin{array}{l}\text { The effects of health on } \\
\text { the ability to work }\end{array}$} & Non-tortured & 379 & 379 & 1.72 & 1.310 & \multirow{2}{*}{-.662} & \multirow{2}{*}{-5.27} & \multirow{2}{*}{.000} & \multirow{2}{*}{.50} \\
\hline & Torture Survivors & 123 & 123 & 2.38 & 1.177 & & & & \\
\hline \multirow{2}{*}{$\begin{array}{l}\text { The effects of health on } \\
\text { memory }\end{array}$} & Non-tortured & 379 & 379 & 1.42 & 1.256 & \multirow{2}{*}{-.472} & \multirow{2}{*}{-3.78} & \multirow{2}{*}{.000} & \multirow{2}{*}{.38} \\
\hline & Torture Survivors & 123 & 123 & 1.89 & 1.186 & & & & \\
\hline \multirow{2}{*}{ Have health problem/s } & Non-tortured & 379 & 379 & .34 & .497 & \multirow{2}{*}{-.299} & \multirow{2}{*}{-5.94} & \multirow{2}{*}{.000} & \multirow{2}{*}{.59} \\
\hline & Torture Survivors & 123 & 123 & .64 & .481 & & & & \\
\hline \multirow{2}{*}{$\begin{array}{l}\text { Have blood pressure } \\
\text { and heart problems }\end{array}$} & Non-tortured & 379 & 379 & .08 & .266 & \multirow{2}{*}{-.130} & \multirow{2}{*}{-4.07} & \multirow{2}{*}{.000} & \multirow{2}{*}{.43} \\
\hline & Torture Survivors & 123 & 121 & .21 & .407 & & & & \\
\hline \multirow{2}{*}{$\begin{array}{l}\text { Have Neurological } \\
\text { problems }\end{array}$} & Non-tortured & 379 & 379 & .07 & .262 & \multirow{2}{*}{-.124} & \multirow{2}{*}{-3.96} & \multirow{2}{*}{.000} & \multirow{2}{*}{.41} \\
\hline & Torture Survivors & 123 & 121 & .20 & .400 & & & & \\
\hline \multirow{2}{*}{$\begin{array}{l}\text { Have Respiratory } \\
\text { problems }\end{array}$} & Non-tortured & 379 & 379 & .06 & .229 & \multirow{2}{*}{-.159} & 524 & 000 & 52 \\
\hline & Torture Survivors & 123 & 121 & .21 & .432 & & -3.24 & .000 & . \\
\hline Have Digestive system & Non-tortured & 379 & 379 & .08 & .278 & & & 160 & 09 \\
\hline problems & Torture Survivors & 123 & 121 & .13 & .340 & .040 & -1.40 & .102 & .02 \\
\hline Have reproductive & Non-tortured & 379 & 378 & .06 & .295 & & & 052 & 23 \\
\hline system problems & Torture Survivors & 123 & 121 & .12 & .331 & .000 & 1.00 & .002 & J \\
\hline Have Musculoskeletal & Non-tortured & 379 & 379 & .14 & .350 & -220 & -568 & 000 & 57 \\
\hline system problem & Torture Survivors & 123 & 121 & .37 & .485 & & & & \\
\hline Have Diabetes/ & Non-tortured & 379 & 379 & .06 & .239 & 121 & 200 & 000 & 10 \\
\hline endocrinology problem & Torture Survivors & 123 & 121 & .18 & .428 & & -3.92 & .000 & .40 \\
\hline Othor bolth noble & Non-tortured & 379 & 379 & .04 & .326 & & & 202 & 00 \\
\hline & Torture Survivors & 123 & 121 & .07 & .346 & & & . & .08 \\
\hline
\end{tabular}

tortured and non-tortured in re-experiencing or avoidance, but the tortured are higher in arousal and especially in dissociation. For PCTD symptom clusters, while there are no significant differences between the two groups in the anxiety/depression/somatization comorbidity cluster, the tortured group is significantly higher in the dissociation-psychoticism cluster, suicidality cluster, and executive control cluster than other Syrian non-tortured refugees. However, there are no significant differences between tortured and non-tortured Syrian refugees in posttraumatic growth, regardless of these other severe health and mental health differences. Further, torture survivors have higher identity salience (group identity) than non-tortured (see Table 2).

Correlational Results: Torture was not associated with PTSD re-experiencing 
I. Kira et al.

Table 2. Independent samples t-test between tortured and non-tortured in mental health, identity salience, and PTG.

\begin{tabular}{|c|c|c|c|c|c|c|c|c|c|}
\hline Variable & Groups & $\mathrm{N}$ & Mean & $\mathrm{SD}$ & SE & $\begin{array}{c}\text { Mean } \\
\text { difference }\end{array}$ & $t$ & $p$ & $\begin{array}{l}\text { Cohen's d } \\
\text { effect size }\end{array}$ \\
\hline \multirow{2}{*}{ Negative Appraisal } & Non-tortured & 379 & 13.06 & 10.07 & .52 & \multirow{2}{*}{-7.58} & \multirow{2}{*}{-6.88} & \multirow{2}{*}{.000} & \multirow{2}{*}{.68} \\
\hline & Torture Survivors & 123 & 20.64 & 12.18 & 1.10 & & & & \\
\hline \multirow{2}{*}{ CSTO } & Non-tortured & 379 & 6.75 & 4.38 & .23 & \multirow{2}{*}{-4.13} & \multirow{2}{*}{-7.97} & \multirow{2}{*}{.000} & \multirow{2}{*}{.84} \\
\hline & Torture Survivors & 123 & 10.88 & 5.18 & .47 & & & & \\
\hline \multirow{2}{*}{ PTG } & Non-tortured & 379 & 55.74 & 19.87 & 1.02 & \multirow{2}{*}{2.18} & \multirow{2}{*}{1.07} & \multirow{2}{*}{.287} & \multirow{2}{*}{.11} \\
\hline & Torture Survivors & 123 & 53.56 & 19.39 & 1.75 & & & & \\
\hline \multirow{2}{*}{ Identity Salience } & Non-tortured & 379 & 19.54 & 8.43 & .43 & \multirow{2}{*}{-1.98} & \multirow{2}{*}{-2.16} & \multirow{2}{*}{.031} & \multirow{2}{*}{.23} \\
\hline & Torture Survivors & 123 & 21.52 & 9.92 & .90 & & & & \\
\hline \multirow{2}{*}{ PTSD } & Non-tortured & 379 & 21.74 & 17.22 & .89 & \multirow{2}{*}{-4.20} & \multirow{2}{*}{-2.37} & \multirow{2}{*}{.019} & \multirow{2}{*}{.24} \\
\hline & Torture Survivors & 123 & 25.93 & 16.99 & 1.53 & & & & \\
\hline \multirow{2}{*}{ PTSD Re-experiencing } & Non-tortured & 379 & 8.09 & 6.79 & .35 & \multirow{2}{*}{-.32} & \multirow{2}{*}{-.49} & \multirow{2}{*}{.645} & \multirow{2}{*}{.05} \\
\hline & Torture Survivors & 123 & 8.41 & 6.15 & .56 & & & & \\
\hline DTSD A moidance & Non-tortured & 379 & 4.55 & 4.07 & .21 & 10 & 40 & & \\
\hline & Torture Survivors & 123 & 4.75 & 3.75 & .34 & & & & \\
\hline PTSD A roucal & Non-tortured & 379 & 4.63 & 4.75 & .25 & $102+2$ & & & \\
\hline & Torture Survivors & 123 & 6.45 & 4.68 & .43 & & & & \\
\hline DTCD Dicrocintion & Non-tortured & 379 & 4.45 & 4.63 & .24 & & 270 & 000 & 10 \\
\hline & Torture Survivors & 123 & 6.34 & 4.99 & .45 & & & & \\
\hline DCTD & Non-tortured & 379 & 19.96 & 11.746 & .61 & & 202 & 000 & .41 \\
\hline & Torture Survivors & 123 & 24.82 & 12.28 & 1.11 & & & & \\
\hline PCTD-Depression/ & Non-tortured & 379 & 5.41 & 2.92 & .151 & & & & \\
\hline $\begin{array}{l}\text { Anxiety/somatization } \\
\text { comorbidity cluster }\end{array}$ & Torture Survivors & 123 & 5.81 & 4.59 & .411 & -.40 & -.90 & .369 & .12 \\
\hline PCTD-Executive & Non-tortured & 379 & 3.58 & 2.83 & .159 & & & 证 & \\
\hline control cluster & Torture Survivors & 123 & 4.49 & 2.72 & .25 & & & & \\
\hline PCTD-Psychoticism- & Non-tortured & 379 & 3.01 & 2.88 & .15 & 126 & 141 & 000 & 16 \\
\hline Dissociation cluster & Torture Survivors & 123 & 4.37 & 3.14 & .29 & & -4.71 & .000 & .40 \\
\hline PCTD-Suicidality & Non-tortured & 379 & 2.12 & 2.83 & .15 & & -328 & 001 & 35 \\
\hline cluster & Torture Survivors & 123 & 3.11 & 3.05 & .28 & & & .000 & .0 \\
\hline
\end{tabular}

or avoidance but was significantly associated with PTSD dissociation (.17, $P$ $<.001)$ and PTSD arousal $(.16, P<.001)$. It was not associated with PCTD anxiety/depression/somatization comorbidity cluster, but it was associated with PCTD-dissociation/psychoticism cluster (.19, $P<.001)$, PCTD-suicidality cluster (.15, $P<.001)$, and PCTD-executive control deficits cluster $(.14, P<.001)$. It was associated with PCTD $(.17, P<.001)$ and PTSD $(.11, P<.05)$. PTSD dissociation 
was highly associated with PCTD-dissociation/psychoticism $(.51, P<.001)$, and PCTD $(.58, P<.001)$. PTSD and PCTD are highly correlated $(.62, P<.001)$. Table 3 includes the zero-order correlation between the variables.

\section{Hierarchical Multiple Regression Results:}

For P-CTD as a dependent variable, in the first step, education was predictive of lower P-CTSD, while marital status (being widowed, divorced) was predictive of higher P-CTD. In the second step, the added Syrian civil war trauma was predictive of higher PCTD. In the last step, when we added torture and intersected discrimination, while education and marital status continued to be significant predictors, Syrian civil war trauma lost its significance as a predictor, while torture and intersected discrimination were significant predictors of higher P-CTSD with torture has relatively larger effect size.

For PTSD, as a dependent variable, no demographic variable was predictive of PTSD in the first step. In the second step, the added Syrian Civil war Trauma did not predict PTSD. In the third step, adding torture and intersected discrimination, only intersected discrimination (and not torture) predicted PTSD. We can conclude that while torture survivors have several traumas, such as intersected discrimination that may be associated with PTSD, their torture trauma is too severe that may be associated with the more severe complex symptoms of P-CTD. Table 4 presents these results.

For the effects of identity salience on PTG, income was a significant predictor of PTG in the first step. In the second step, no demographic variable was predictive of PTG; identity salience predicted PTG with a relatively high effect size $(\beta=.51)$. Table 5 presents these results. We can conclude that identity salience is a critical variable in predicting posttraumatic growth in torture survivors.

Table 3. Zero-order correlations between torture and PTSD and P-CTD symptom clusters.

\begin{tabular}{|c|c|c|c|c|c|c|c|c|c|c|c|}
\hline Variable & 1 & 2 & 3 & 4 & 5 & 6 & 7 & 8 & 9 & 10 & 11 \\
\hline 1. Torture & 1 & & & & & & & & & & \\
\hline 2. PTSD-Rexperiencing & .02 & 1 & & & & & & & & & \\
\hline 3. PTSD-Avoidance & .021 & $.81^{\star \star \star}$ & 1 & & & & & & & & \\
\hline 4. PTSD-Arousal & $.16^{\star * *}$ & $.56^{\star * *}$ & $.55^{\star * *}$ & 1 & & & & & & & \\
\hline 5. PTSD-Dissociation & $.17^{\star \star *}$ & $.62^{\star * \star}$ & $.66^{* * *}$ & $.65^{\star \star *}$ & 1 & & & & & & \\
\hline 6.PCTD-Executive control deficits & $.14^{\star * *}$ & $.37^{\star * *}$ & $.37^{\star * *}$ & $.39^{* * *}$ & $.39^{* * *}$ & 1 & & & & & \\
\hline 7. PCTD-Psychoticism/dissociation & $.19^{* * *}$ & $.36^{* * *}$ & $.34^{\star * *}$ & $.53^{* * *}$ & $.51^{* * *}$ & $.49^{* * *}$ & 1 & & & & \\
\hline 8. PCTD-Suicidality & $.15^{\star \star \star}$ & $.29^{\star * *}$ & $.28^{\star * *}$ & $.53^{\star * *}$ & $.43^{* * *}$ & $.38^{\star * *}$ & $.57^{\star \star *}$ & 1 & & & \\
\hline $\begin{array}{l}\text { 9. PCTD-Depression/anxiety/ } \\
\text { somatization comorbidity }\end{array}$ & .05 & $.40^{\star * *}$ & $.32^{\star * *}$ & $.250^{* * *}$ & $.30^{\star * *}$ & $.48^{\star * *}$ & $.33^{\star * *}$ & $.20^{* * *}$ & 1 & & \\
\hline 10.PCTD & $.17^{\star \star \star *}$ & $.52^{\star \star *}$ & $.49^{\star * *}$ & $.53^{\star * *}$ & $.58^{\star \star \star}$ & $.74^{\star * *}$ & $.77^{\star \star \star}$ & $.68^{* * *}$ & $.67^{\star * *}$ & 1 & \\
\hline 11. PTSD & $.11^{\star}$ & $.90^{\star \star \star *}$ & $.88^{\star \star \star *}$ & $.80^{\star \star \star}$ & $.85^{\star \star \star}$ & $.44^{\star \star * *}$ & $.50^{\star * \star}$ & $.44^{\star \star \star *}$ & $.38^{* * *}$ & $.62^{* * *}$ & 1 \\
\hline
\end{tabular}

Note: ${ }^{\star} p<.05,{ }^{* *} p<.01,{ }^{* *} p<.001$ (two-tailed). Note: PTSD = Postraumatic stress disorder, PCTD = Post-cumulative trauma disorders. 
I. Kira et al.

Table 4. Hierarchical multiple regression for the effects of torture, intersected discrimination and Syrian civil war traumas on PTSD and complex.

\section{The Effects of Torture and Intersected discrimination on complex PTSD (Post-cumulative traumatic stress disorder)}

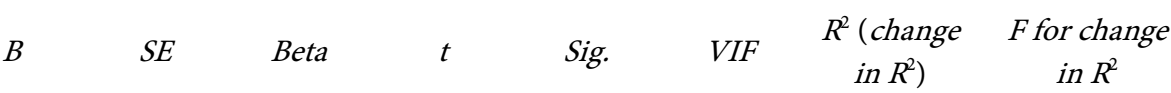

\section{Model 1: Step One}

\begin{tabular}{|c|c|c|c|c|c|c|c|c|}
\hline Gender & -1.669 & 1.141 & -.065 & -1.463 & .144 & 1.046 & \multirow{5}{*}{.061} & \multirow{5}{*}{$6.394(<.000)$} \\
\hline Age & -.004 & .049 & -.003 & -.077 & .939 & 1.069 & & \\
\hline Marital Status & 2.196 & .603 & .169 & 3.640 & .000 & 1.135 & & \\
\hline Education & -1.202 & .394 & -.161 & -3.050 & .002 & 1.464 & & \\
\hline Yearly income & .227 & .348 & .033 & .654 & .514 & 1.342 & & \\
\hline \multicolumn{9}{|l|}{ Model 2: Step Two } \\
\hline Gender & -1.314 & 1.147 & -.051 & -1.145 & .253 & 1.067 & \multirow{6}{*}{.009} & \multirow{6}{*}{$4.942(<.027)$} \\
\hline Age & -.020 & .049 & -.018 & -.399 & .690 & 1.092 & & \\
\hline Marital Status & 2.215 & .601 & .170 & 3.685 & .000 & 1.135 & & \\
\hline Education & -1.187 & .393 & -.159 & -3.023 & .003 & 1.465 & & \\
\hline Yearly income & .218 & .346 & .032 & .630 & .529 & 1.342 & & \\
\hline Syrian Civil war Trauma & .053 & .024 & .099 & 2.223 & .027 & 1.048 & & \\
\hline \multicolumn{9}{|l|}{ Model 3: Step Three } \\
\hline Gender & -.333 & 1.167 & -.013 & -.285 & .775 & 1.128 & \multirow{8}{*}{.024} & \multirow{8}{*}{$6.528(<.002)$} \\
\hline Age & -.020 & .049 & -.018 & -.404 & .686 & 1.102 & & \\
\hline Marital Status & 2.064 & .598 & .159 & 3.450 & .001 & 1.149 & & \\
\hline Education & -1.162 & .388 & -.155 & -2.990 & .003 & 1.465 & & \\
\hline Yearly income & .523 & .353 & .076 & 1.482 & .139 & 1.424 & & \\
\hline Syrian Civil war Trauma & .029 & .028 & .054 & 1.033 & .302 & 1.461 & & \\
\hline Intersected discriminations & 1.349 & .645 & .097 & 2.093 & .037 & 1.178 & & \\
\hline Torture & 3.525 & 1.502 & .127 & 2.346 & .019 & 1.583 & & \\
\hline
\end{tabular}

The Effects of Torture and Intersected discrimination on PTSD

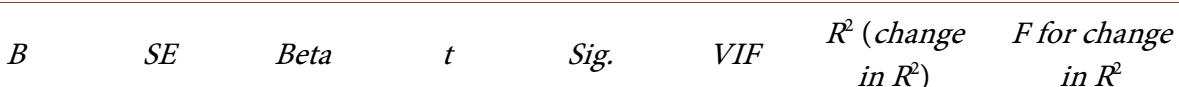

\section{Model 1: Step One}

\begin{tabular}{|c|c|c|c|c|c|c|c|c|}
\hline Gender & -1.535 & 1.676 & -.042 & -.916 & .360 & 1.046 & \multirow{5}{*}{.019} & \multirow{5}{*}{$1.938(<.087)$} \\
\hline Age & .063 & .072 & .040 & .880 & .379 & 1.069 & & \\
\hline Marital Status & 1.695 & .887 & .091 & 1.911 & .057 & 1.135 & & \\
\hline Education & -.915 & .579 & -.085 & -1.580 & .115 & 1.464 & & \\
\hline Yearly income & .215 & .511 & .022 & .421 & .674 & 1.342 & & \\
\hline \multicolumn{9}{|c|}{ Model 2: Step Two } \\
\hline Gender & -1.366 & 1.693 & -.037 & -.807 & .420 & 1.067 & \multirow[t]{2}{*}{.001} & \multirow[t]{2}{*}{$.516(<.473)$} \\
\hline Age & .056 & .073 & .036 & .766 & .444 & 1.092 & & \\
\hline
\end{tabular}




\section{Continued}

\begin{tabular}{|c|c|c|c|c|c|c|c|c|}
\hline Marital Status & 1.703 & .887 & .091 & 1.920 & .055 & 1.135 & & \\
\hline Education & -.908 & .580 & -.084 & -1.567 & .118 & 1.465 & & \\
\hline Yearly income & .211 & .511 & .021 & .412 & .680 & 1.342 & & \\
\hline Syrian Civil war Trauma & .025 & .035 & .033 & .718 & .473 & 1.048 & & \\
\hline \multicolumn{9}{|l|}{ Model 3: Step Three } \\
\hline Gender & .077 & 1.715 & .002 & .045 & .964 & 1.128 & \multirow{8}{*}{.033} & \multirow{8}{*}{$8.516(<.000)$} \\
\hline Age & .068 & .072 & .044 & .946 & .345 & 1.102 & & \\
\hline Marital Status & 1.338 & .880 & .071 & 1.521 & .129 & 1.149 & & \\
\hline Education & -.887 & .571 & -.082 & -1.553 & .121 & 1.465 & & \\
\hline Yearly income & .646 & .519 & .065 & 1.246 & .213 & 1.424 & & \\
\hline Syrian Civil war Trauma & .025 & .041 & .032 & .610 & .542 & 1.461 & & \\
\hline Intersected discriminations & 3.458 & .948 & .174 & 3.649 & .000 & 1.178 & & \\
\hline Torture & 2.162 & 2.209 & .054 & .979 & .328 & 1.583 & & \\
\hline
\end{tabular}

Table 5. Hierarchical multiple regression for the effects of identity salience on posttraumatic growth.

The Effects of Identity Salience on PTG

$\begin{array}{llllccc}R^{2} \text { (change } & \text { F for change } \\ \left.\text { in } R^{2}\right) & \text { in } R^{2}\end{array}$

\section{Model 1: Step One}

\begin{tabular}{|c|c|c|c|c|c|c|c|c|}
\hline Gender & .386 & 1.964 & .009 & .197 & .844 & 1.049 & \multirow{5}{*}{.023} & \multirow{5}{*}{$3.25(<.007)$} \\
\hline Age & -.057 & .085 & -.031 & -.671 & .502 & 1.076 & & \\
\hline Marital Status & .648 & 1.033 & .030 & .627 & .531 & 1.147 & & \\
\hline Education & .107 & .680 & .009 & .158 & .875 & 1.473 & & \\
\hline Yearly income & -2.009 & .595 & -.176 & -3.379 & .001 & 1.349 & & \\
\hline \multicolumn{9}{|c|}{ Model 2: Step Two } \\
\hline Gender & .735 & 1.725 & .017 & .426 & .670 & 1.050 & \multirow{6}{*}{.222} & \multirow{6}{*}{$143.92(<.000)$} \\
\hline Age & -.088 & .074 & -.048 & -1.187 & .236 & 1.077 & & \\
\hline Marital Status & .485 & .907 & .023 & .535 & .593 & 1.147 & & \\
\hline Education & 1.101 & .603 & .088 & 1.826 & .068 & 1.501 & & \\
\hline Yearly income & -.504 & .537 & -.044 & -.938 & .349 & 1.427 & & \\
\hline Identity Salience & 1.137 & .095 & .508 & 11.997 & .000 & 1.162 & & \\
\hline
\end{tabular}

\section{Conclusion and Discussion}

The current study is significant, as it did not only replicate previous studies' results on the effects of torture on PTSD and other mental health conditions and the relatively higher or comparable rates of PTG, compared to non-tortured but also on identifying some of the dynamics and mechanisms behind these relationships. The study confirmed the study hypotheses and replicated and expanded 
on previous studies' results. Syrian torture survivors were exposed to more traumatic experiences than other Syrian refugees who were not tortured. They suffered more symptoms of PTSD and P-CTD compared to other non-tortured refugees. They endured more physical health problems, primarily neurological and musculoskeletal, consistent with their torture experience. Neurological problems may be associated with memory and executive function deficits. They were higher in arousal, dissociation, psychoticism-dissociation, executive dyscontrol, and suicidality symptoms. However, regardless, as hypothesized, there were no significant differences between them and non-tortured in their post-traumatic growth, and they had higher identity salience (group identity) than non-tortured, which seems to be a protective factor for them as will be discussed further. While torture trauma was not associated with re-experiencing or avoidance symptoms, it was significantly associated with PTSD-dissociation, PTSD arousal, P-CTD psychoticism-dissociation, P-CTD poor executive control, and suicidality. In the hierarchical multiple regressions, torture was the strong predictor of P-CTD, while it did not predict PTSD, supporting previous findings on the effects of torture (Hollifield et al., 2011, Kira et al., 2006). Intersected discrimination predicted PTSD in torture survivors and refugees. To conclude, torture trauma may be too severe as it was associated with the more severe complex symptoms of P-CTD, rather than only PTSD symptoms. PTSD in torture survivors may be more related to other stressors and trauma experiences (e.g., intersected discrimination, cumulative stressors, and traumas dynamics).

A previous study on Iraqi refugees found that torture survivors are higher in PTG than other non-tortured refugees (Kira et al., 2006). Current results did not find higher PTG in Syrian torture survivors compared to the non-tortured but found comparable rates, though they have more trauma and symptom overloads than their counterparts. PTG in tortured refugees is enhanced by higher group (collective) identity salience, as the current study indicated, which is the mechanism behind their higher or comparable PTG.

Identity salience predicted PTG with a relatively high effect $(\beta=.51)$. Identity salience was significantly higher in torture survivors than non-tortured refugees, making sense for those who had sacrificed themselves for their group identity and or for its cause. The current study might help elucidate the detrimental effect of the unattainability of positive group identity for minority groups who suffer discrimination, marginalization, and the belittling of their group identity, and those victims/survivors of sex trafficking, Satanic cults, and other criminal enterprises. A related question is whether, for the positive effect of group identity, the identity must precede or be enhanced by the torture, or whether it can be efficacious or feasible if developed in a post-refuge context after the torture or through intervention and prevention strategies.

The current study has significant conceptual and clinical implications. Conceptually, psychiatric nosologies provided various diagnostic categories that can cluster into profiles and syndromes following varieties of severe adversities and contingencies. Clinicians and clinical scientists instead adopt the primacy of pro- 
files of syndromes that follow severe traumas over specific PTSD or C-PTSD diagnostic categories to correctly identify and effectively treat their mental and cognitive negative impact on the individuals and groups. Post-cumulative trauma dynamics and reactions may be far greater than a single diagnostic category, especially for torture survivors and refugees. Clinical science may better be served by focusing more on the pathways of such syndromes and profiles and the varieties of their trajectories over time. Network analysis of DSM structure indicated that psychopathology could be more adequately captured by sophisticated network models than current classification systems (Boschloo et al., 2015). However, focusing on empirically identified syndromes and trajectories is not to replace diagnostic categories but rather to expand the frontiers and precision of clinical science into new directions that are more consistent with clinical phenomena and their lived realities.

The study results have important clinical implications for intervention and prevention, working with torture survivors. The importance of the identity variable, especially when dealing with torture survivors, seems a critical variable that is neglected in most therapeutic approaches with refugees and torture survivors. The identity variable is pre-cognitive and related to the executive self.

Another critical variable is intersected discrimination that torture survivors and refugees encounter in their native and host countries. The transnational intersectionality framework was proposed to extend the intersectionality perspective, which may help better understand the complexities of power and oppression across national contexts and their influence on torture survivors' lives (Gangamma \& Shipman, 2018). Narrative identity therapy that may help to empower/re-empower transform, re-author, and reauthorize identity with narrative techniques may be one of the keys for healing for torture survivors and enhancing PTG (Kira \& Tummala-Narra, 2015; Kira et al., 2015; Kira et al., 2012a; Kira et al., 2014; Kira, 2002; McAdams \& Janis, 2004; Rousseau et al., 2005).

The effects of torture on dissociation and executive control function are alarming and need a particular focus on treatment. A recent study confirmed the direct effects of torture on higher working memory and inhibition deficits, in addition to its indirect effects on them via mental health symptoms (Kira et al., 2020b; Kira et al., paper submitted for publication). There is a pressing need for cognitive training to enhance their executive functions, critical to their mental health treatment. Several studies have reported cognitive training efficacy in improving cognitive control (e.g., Han, Chapman, \& Krawczyk, 2018). Such cognitive interventions should be part of the standard treatment protocol for treating torture survivors and may be essential for PTSD and depression treatment protocols. Innovations and experimentation on cognitive training with torture survivors and refugees are desperately needed. Additionally treatment should include secondary torture survivors (e.g., family members) (e.g., Kira, 2004). 


\section{Limitations and Future Direction}

The current study has several limitations. One of the limitations is that the study was conducted on a convenient sample with narrow and biased representation. Another limitation is that the measures used are based on self-reports, which are subject to over or under-reporting due to social desirability. Another weakness is that the study utilized a cross-sectional design.

However, the current study provided significant replication and extension of previous findings on the nature of torture trauma and its effects and the need for novel rehabilitation and treatment protocols for torture survivors, including unique applications and some key active treatment ingredients, such as identity-focused interventions and cognitive training.

\section{Compliance with Ethical Standards}

The study is a secondary analysis. The original data was collected upon approval of the IRB of the Fayoum University, EGYPT, and the studies were performed in accordance with the ethical standards as set forth in the 1964 Declaration of Helsinki and its later amendments.

\section{Informed Consent}

The study is a secondary analysis. Informed consent was obtained in the original studies

\section{Conflicts of Interest}

The authors declare they have no conflict of interest.

\section{References}

Abu Suhaiban, H., Grasser, L. R., \& Javanbakht, A. (2019). Mental Health of Refugees and Torture Survivors: A Critical Review of Prevalence, Predictors, and Integrated Care. International Journal of Environmental Research and Public Health, 16, Article No. 2309. https://doi.org/10.3390/ijerph16132309

Al-Ibraheem, B., Kira, I., Aljakoub, J., \& Al-Ibraheem, A. (2017). The Health Effect of the Syrian Conflict on IDPs and Refugees. Peace and Conflict: Journal of Peace Psychology, 23, 140-152. https://doi.org/10.1037/pac0000247

Betemps, E. J., Smith, R. M., Baker, D. G., \& Rounds-Kugler, B. A. (2003). Measurement Precision of the Clinician Administered PTSD Scale (CAPS): A RASCH Model Analysis. Journal of Applied Measurement, 4, 59-69.

Boschloo, L., van Borkulo, C. D., Rhemtulla, M., Keyes, K. M., Borsboom, D., \& Schoevers, R. A. (2015). The Network Structure of Symptoms of the Diagnostic and Statistical Manual of Mental Disorders. PLoS One, 10, e0137621. https://doi.org/10.1371/journal.pone.0137621

Choi, H., Lee, H. J., \& Lee, H. Y. (2017). The Effects of Torture-Related Stressors on Long-Term Complex Post-Traumatic Symptoms in South Korean torture survivors. International Journal of Psychology, 52, 57-66. https://doi.org/10.1002/ijop.12276

Chu, T., Keller, A. S., \& Rasmussen, A. (2013). Effects of Post-Migration Factors on PTSD Outcomes among Immigrant Survivors of Political Violence. Journal of Immigrant and 
Minority Health, 15, 890-897. https://doi.org/10.1007/s10903-012-9696-1

Cohen, J. (1992). A Power Primer. Psychological Bulletin, 112, 155-159. https://doi.org/10.1037/0033-2909.112.1.155

Connor, P., \& Krogstadl, J. M. (2016). About Six in ten Syrians Are Now Displaced from Their Homes. PEW Research Center.

http://www.pewresearch.org/fact-tank/2016/06/13/about-six-in-tensyrians-are-now-dis placed-from-their-homes/

Edge, S., \& Newbold, B. (2013). Discrimination and the Health of Immigrants and Refugees: Exploring Canada's Evidence Base and Directions for Future Research in Newcomer Receiving Countries. Journal of Immigrant and Minority Health, 15, 141-148. https://doi.org/10.1007/s10903-012-9640-4

Elmore, K. C., \& Oyserman, D. (2012). If 'We' Can Succeed, 'I' Can Too: Identity-Based Motivation and Gender in the Classroom. Contemporary Educational Psychology, 37, 176-185. https://doi.org/10.1016/j.cedpsych.2011.05.003

Gangamma, R., \& Shipman, D. (2018). Transnational Intersectionality in Family Therapy with Resettled Refugees. Journal of Marital and Family Therapy, 44, 206-219. https://doi.org/10.1111/jmft.12267

Hair, J. F., Hult, G. T. M., Ringle, C. M., \& Sarstedt, M. (2017). A Primer on Partial Least Squares Structural Equation Modeling (PLS-SEM) (2nd ed.). Sage.

Han, K., Chapman, S. B., \& Krawczyk, D. C. (2018). Neuroplasticity of Cognitive Control Networks Following Cognitive Training for Chronic Traumatic Brain Injury. NeuroImage: Clinical, 18, 262-278. https://doi.org/10.1016/j.nicl.2018.01.030

Helgeson, V. S., Reynolds, K. A., \& Tomich, P. L. (2006). A Meta-Analytic Review of Benefit Finding and Growth. Journal of Consulting and Clinical Psychology, 74, 797-816. https://doi.org/10.1037/0022-006X.74.5.797

Hollifield, M., Warner, T. D., \& Westermeyer, J. (2011). Is Torture Reliably Assessed and a Valid Indicator of Poor Mental Health? Journal of Nervous and Mental Disease, 199, 3-10. https://doi.org/10.1097/NMD.0b013e3182051501

Hooberman, J. B., Rosenfeld, B., Lhewa, D., Rasmussen, A., \& Keller, A. (2007). Classifying the torture experiences of refugees living in the United States. Journal of Interpersonal Violence, 22, 108-123. https://doi.org/10.1177/0886260506294999

Johnson, H., \& Thompson, A. (2008). The Development and Maintenance of Post-Traumatic Stress Disorder (PTSD) in Civilian Adult Survivors of War Trauma and Torture: A Review. Clinical Psychology Review, 28, 36-47. https://doi.org/10.1016/j.cpr.2007.01.017

Kalt, A., Hossain, M., Kiss, L., \& Zimmerman, C. (2013). Asylum Seekers, Violence, and Health: A Systematic Review of Research in High-Income Host Countries. American Journal of Public Health, 103, e30-e42. https://doi.org/10.2105/AJPH.2012.301136

Kanaan, A., Kira, I., Shuwiekh, H., Kucharska, J., \& Al-Huwailah, A. (2019). The Dynamics behind Low Posttraumatic Growth in Victims of Type III Traumas: The Case of Syrians and Palestinians. Traumatology, 26, 205-214. https://doi.org/10.1037/trm0000229

Kim, I., Keovisai, M., Kim, W., Richards-Desai, S., \& Yalim, A. C. (2019). Trauma, Discrimination, and Psychological Distress across Vietnamese Refugees and Immigrants: A Life Course Perspective. Community Mental Health Journal, 55, 385-393. https://doi.org/10.1007/s10597-018-0268-2

Kira, I. (2001). Taxonomy of Trauma and Trauma Assessment. Traumatology, 7, 73-86. https://doi.org/10.1177/153476560100700202

Kira, I. (2002). Torture Assessment and Treatment: The Wraparound Approach. Traumatology, 8, 54-86. https://doi.org/10.1177/153476560200800203 
Kira, I. (2004). Secondary Trauma in Treating Refugee Survivors of Torture and Their Families. Torture, 14, 38-44.

Kira, I. (2010). Etiology and Treatment of Post-Cumulative Traumatic Stress Disorders in Different Cultures. Traumatology, 16, 128-141. https://doi.org/10.1177/1534765610365914

Kira, I. (2017). A Critical Outlook at Torture Definition, Structure, Dynamics, and Interventions. Peace and Conflict: Journal of Peace Psychology, 23, 328-333. https://doi.org/10.1037/pac0000243

Kira, I. (2019). Toward an Integrative Theory of Self-Identity and Identity Stressors and Traumas and Its Mental Health Dynamics. Psychology, 10, 385-410. https://doi.org/10.4236/psych.2019.104027

Kira, I. (2020). Refugee Status and Identity Development. In S. Hupp, \& J. Jewell (Eds.), The Encyclopedia of Child and Adolescent Development. Wiley. https://doi.org/10.1002/9781119171492.wecad467

Kira, I. (2021a). Taxonomy of Stressors and Traumas: An Update of The Development-Based Trauma Framework (DBTF): A life-Course perspective on Stress and Trauma. Traumatology. Advance Online Publication. https://doi.org/10.1037/trm0000305

Kira, I. (2121b). The Development-Based Taxonomy of Stressors and Traumas: An Initial Empirical Validation. Psychology, 12, 1575-1614.

Kira, I. A., \& Shuwiekh, H. (2021). Discrimination and Mental Health of Christians in Egypt: Coping Trajectories and Perceived Posttraumatic Growth. Mental Health, Religion, and Culture, 24, 1-22. https://doi.org/10.1080/13674676.2020.1832454

Kira, I. A., Ibrahim, E. R., Shuwiekh, H., \& Ashby, S. (2021b). Does Intersected Discrimination Underlie the Disproportionate Impact of COVID-19 Infection and Its Severity on Minorities? An Example from Jordan. Journal of Loss and Trauma: International Perspectives on Stress \& Coping. https://doi.org/10.1080/15325024.2021.1992224

Kira, I., \& Tummala-Narra, P. (2015). Psychotherapy with Refugees: Emerging Paradigm. Journal of Loss and Trauma, 20, 449-467. https://doi.org/10.1080/15325024.2014.949145

Kira, I., Abou_Mediene, S., Ashby, J., Lewandowski, L., Mohanesh, J., \& Odenat, L. (2012c). Post-Traumatic Growth Inventory: Psychometric Properties of the Arabic Version in Palestinian Adults. The International Journal of Educational and Psychological Assessment, 11, 120-137.https://doi.org/10.1037/t60436-000

Kira, I., Abou-Mediene, S., Ashby, J., Odenat, L., Mohanesh, J., \& Alamia, H. (2013a). The Dynamics of Post-Traumatic Growth across Different Trauma Types in a Palestinian Sample. Journal of Loss and Trauma, 18, 120-139. https://doi.org/10.1080/15325024.2012.679129

Kira, I., Ahmed, A., Wasim, F., Mahmoud, V., Colrain, J., \& Rai, D. (2012a). Group Therapy for Refugees and Torture Survivors: Treatment Model Innovations. International Journal of Group Psychotherapy, 62, 69-88. https://doi.org/10.1521/ijgp.2012.62.1.69

Kira, I., Alawneh, A. N., Aboumediane, S., Mohanesh, J., Ozkan, B., \& Alamia, H. (2011). Identity Salience and Its Dynamics in Palestinian Adolescents. Psychology, 2, 781-791. https://doi.org/10.4236/psych.2011.28120

Kira, I., Alpay, E., Shuwiekh, H., Ashby, J. S., Turkeli, A., \& Alhuwailah (Paper Submitted for Publication). The Effects of COVID-19 on Torture Survivors' Mental Health and Cognitive Functioning.

Kira, I., Amer, M. M., \& Wrobel, N. H. (2014). Arab Refugees: Trauma, Resilience, and 
Recovery. In S. C. Nassar-McMillan, K. J. Ajrouch, \& J. Hakim-Larson (Eds.), Biopsychosocial Approach to Arab Americans: Perspectives on Culture, Development, and Health (pp. 175-195). Springer. https://doi.org/10.1007/978-1-4614-8238-3_9

Kira, I., Ashby J. S., Lewandowski, L., Alawneh, A. N., Mohanesh, J., \& Odenat, L. (2013b). Advances in Continuous Traumatic Stress Theory: Traumatogenic Dynamics and Consequences of Intergroup Conflict: The Palestinian Adolescents Case. Psychology, 4, 396-409. https://doi.org/10.4236/psych.2013.44057

Kira, I., Ashby, J. S., Odenat, L., \& Lewandowsky, L. (2013c). The Mental Health Effects of Torture Trauma and Its Severity: A Replication and Extension. Psychology, 4, 472-482. https://doi.org/10.4236/psych.2013.45067

Kira, I., Ashby, J. S., Omidy, A. Z., \& Lewandowski, L. (2015). Current, Continuous, and Cumulative Trauma-Focused Cognitive Behavior Therapy: A New Model for Trauma Counseling. Journal of Mental Health Counseling, 37, 323-340. https://doi.org/10.17744/mehc.37.4.04

Kira, I., Barger, B., Shuwiekh, H., Kucharska, J., \& Al-Huwailah, A. (2020a). The Threshold Non-Linear Model for the Effects of Cumulative Stressors and Traumas: A Chained Cusp Catastrophe Analysis. Psychology, 11, 385-403. https://doi.org/10.4236/psych.2020.113025

Kira, I., Barger, B., Shuwiekh, H., Kucharska, J., \& Al-Huwailah, A. H. (2019c). Cumulative Stressors and Traumas and Suicide: A Non-Linear Cusp Dynamic Systems Model. Psychology, 10, 1999-2018. https://doi.org/10.4236/psych.2019.1015128

Kira, I., Clifford, D., Wiencek, P., \& Al-Haidar, A. (2001). Iraqi Refugees in Southeast Michigan: First Report. ACCESS Community Health and Research Center.

Kira, I., Fawzi, M., \& Fawzi, M. (2013d). The Dynamics of Cumulative Trauma and Trauma Types in Adult Patients with Psychiatric Disorders: Two Cross-Cultural Studies. Traumatology, 19, 179-195. https://doi.org/10.1177/1534765612459892

Kira, I., Fawzi, M., Shuwiekh, H., Lewandowski, L., Ashby, J., \& Al Ibraheem, B. (2021a). Do Adding Attachment, Oppression, Cumulative, and Proliferation Trauma Dynamics to PTSD Criterion "A" Improve Its Predictive Validity: Toward a Paradigm Shift? Current Psychology, 40, 2665-2679. https://doi.org/10.1007/s12144-019-00206-Z

Kira, I., Lewandowski, L., Templin, T., Ramaswamy, V., Ozkan, B., \& Mohanesh, J. (2008). Measuring Cumulative Trauma Dose, Types, and Profiles Using a Development-Based Taxonomy of Traumas. Traumatology, 14, 62-87.

https://doi.org/10.1177/1534765608319324

Kira, I., Lewandowski, L., Templin, T., Ramaswamy, V., Ozkan, B., \& Mohanesh, J. (2010). The Effects of Perceived Discrimination and Backlash on Iraqi Refugees' Physical and Mental Health. Journal of Muslim Mental Health, 5, 59-81.

https://doi.org/10.1080/15564901003622110

Kira, I., Shuwiekh, H., Al Ibraheem, B., \& Aljakoub, J. (2019b). Appraisals and Emotion Regulation Mediate the Effects of Identity Salience and Cumulative Stressors and Traumas, on PTG and Mental Health: The Case of Syrian's IDPs and Refugees. Self and Identity: The Journal of the International Society for Self and Identity, 18, 284-305. https://doi.org/10.1080/15298868.2018.1451361

Kira, I., Shuwiekh, H., Al-Huwailah, A. H., Lewandowski, L., Alawneh, A.-W. N., Abou-Mediene, S., Al Ibraheem, B., \& Aljakoub, J. (2019a). The Central Role of Social Identity in Oppression, Discrimination, and Social-Structural Violence: Collective Identity Stressors and Traumas, Their Dynamics and Mental Health Impact. Peace and Conflict: Journal of Peace Psychology, 25, 262-268. https://doi.org/10.1037/pac0000363

Kira, I., Shuwiekh, H., Al-Huwailah, A., Elwakeel, S., Waheep, N., Ebada, E., \& Ibrahim, 
E. (2020b). The Direct and Indirect Impact of Trauma Types and Cumulative Stressors and Traumas on Executive Functions. Journal of Applied Neuropsychology: Adult. Advance Online First. https://doi.org/10.1080/23279095.2020.1848835

Kira, I., Shuwiekh, H., Kucharska, J., Fawzi, M., Ashby, J. S., Omidy, A. Z., Abou-Mediene, S., \& Lewandowski, L. (2018). Trauma Proliferation and Stress Generation (TPSG) Dynamics and Their Implications for Clinical Science. American Journal of Orthopsychiatry, 88, 582-596. https://doi.org/10.1037/ort0000304

Kira, I., Shuwiekh, H., Rice, K., Al Ibraheem, B., \& Aljakoub, J. (2017). A Threatened Identity: The Mental Health Status of Syrian Refugees in Egypt and Its Etiology. Identity: An International Journal of Theory and Research, 17, 176-190. https://doi.org/10.1080/15283488.2017.1340163

Kira, I., Templin, T., Lewandowski, L., Ashby, J. S., Oladele, A., \& Odenat, L. (2012b). Cumulative Trauma Disorder Scale (CTD): Two Studies. Psychology, 3, 643-656. https://doi.org/10.4236/psych.2012.39099

Kira, I., Templin, T., Lewandowski, L., Clifford, D., Wiencek, E., Hammad, A., Mohanesh, J., \& Al-Haidar, A.-M. (2006). The Effects of Torture: Two Community Studies. Peace and Conflict: Journal of Peace Psychology, 12, 205-228. https://doi.org/10.1207/s15327949pac1203_1

Kleim, B., \& Ehlers, A. (2009). Evidence for a Curvilinear Relationship between Posttraumatic Growth and Posttrauma Depression and PTSD in Assault Survivors. Journal of Traumatic Stress, 22, 45-52. https://doi.org/10.1002/jts.20378

Mayr, S., Erdfelder, E., Buchner, A., \& Faul, F. (2007). A Short Tutorial of G Power. Tutorials in Quantitative Methods for Psychology, 3, 51-59. https://doi.org/10.20982/tqmp.03.2.p051

McAdams, D. P., \& Janis, L. (2004). Narrative Identity and Narrative Therapy. In L. E. Angus \& J. McLeod (Eds.), The Handbook of Narrative and Psychotherapy: Practice, Theory, and Research (pp. 159-173). Sage Publications, Inc. https://doi.org/10.4135/9781412973496.d13

Moreno, A., \& Grodin, M. A. (2002). Torture and Its Neurological Sequelae. Spinal Cord, 40, 213-223. https://doi.org/10.1038/sj.sc.3101284

Mossakowski, K. N. (2003). Coping with Perceived Discrimination: Does Ethnic Identity Protect Mental Health? Journal of Health and Social Behavior, 44, 318-331. https://doi.org/10.2307/1519782

Nicholson, B. L. (1997). The Influence of Pre-Emigration and Postemigration Stressors on Mental Health: A Study of Southeast Asian Refugees. Social Work Research, 21, 19-31. https://doi.org/10.1093/swr/21.1.19

Olsen, D. R., Montgomery, E., Bøjholm, S., \& Foldspang, A. (2006). Prevalent Musculoskeletal Pain as a Correlate of Previous Exposure to Torture. Scandinavian Journal of Public Health, 34, 496-503. https://doi.org/10.1080/14034940600554677

Rathke, H., Poulsen, S., Carlsson, J., \& Palic, S. (2020). PTSD with Secondary Psychotic Features among Trauma-Affected Refugees: The Role of Torture and Depression. Psychiatry Research, 287, Article ID: 112898. https://doi.org/10.1016/j.psychres.2020.112898

Rousseau, C., Gauthier, M. F., Lacroix, L., Alain, N., Benoit, M., Moran, A. et al. (2005). Playing with Identities and Transforming Shared Realities: Drama Therapy Workshops for Adolescent Immigrants and Refugees. The Arts in Psychotherapy, 32, 13-27. https://doi.org/10.1016/j.aip.2004.12.002

Shue, H. (1978). Torture. Philosophy and Public Affairs, 7, 124-143. 
http://www.jstor.org/stable/2264988.

Silove, D. (1999). The Psychosocial Effects of Torture, Mass Human Rights Violations, and Refugee Trauma: Toward an Integrated Conceptual Framework. Journal of Nervous and Mental Disease, 187, 200-207. https://doi.org/10.1097/00005053-199904000-00002

Smith, T. B., \& Silva, L. (2011). Ethnic Identity and Personal Well-Being of People of Color: A Meta-Analysis. Journal of Counseling Psychology, 58, 42-60. https://doi.org/10.1037/a0021528

Song, S. J., Subica, A., Kaplan, C., Tol, W., \& de Jong, J. (2018). Predicting the Mental Health and Functioning of Torture Survivors. The Journal of Nervous and Mental Disease, 206, 33-39. https://doi.org/10.1097/NMD.0000000000000678

Steel, Z., Chey, T., Silove, D., Marnane, C., Bryant, R. A., \& Van Ommeren, M. (2009). Association of Torture and Other Potentially Traumatic Events with Mental Health Outcomes among Populations Exposed to Mass Conflict and Displacement: A Systematic Review and Meta-Analysis. JAMA, 302, 537-549.

https://doi.org/10.1001/jama.2009.1132

Steel, Z., Silove, D., Bird, K., McGorry, P., \& Mohan, P. (1999). Pathways from War Trauma to Posttraumatic Stress Symptoms among Tamil Asylum Seekers, Refugees, and Immigrants. Journal of Traumatic Stress, 12, 421-435. https://doi.org/10.1023/A:1024710902534

Stryker S. (1968). Identity Salience and Role Performance: The Relevance of Symbolic Interaction Theory for Family Research. Journal of Marriage and the Family, 30, 558-564. https://doi.org/10.2307/349494

Summerfield, D. (2003). Fighting “Terrorism” with Torture. British Medical Journal, 326, 773-774. https://doi.org/10.1136/bmj.326.7393.773

Tabachnick, B. G., \& Fidell, L. S. (2013). Using Multivariate Statistics: International Edition. Pearson.

Tedeschi, R. G., \& Calhoun, L. G. (1996). The Posttraumatic Growth Inventory: Measuring the Positive Legacy of Trauma. Journal of Traumatic Stress, 9, 455-471. https://doi.org/10.1002/jts.2490090305

Varvin, S., \& Rosenbaum, B. (2003). Extreme Traumatization: Strategies for Mental Survival. International Forum of Psychoanalysis, 12, 5-16. https://doi.org/10.1080/08037060310005223

Weathers, F. W., Keane, T. M., \& Davidson, J. R. (2001). Clinician-Administered PTSD Scale: A Review of the First Ten Years of Research. Depression and Anxiety, 13, 132-156. https://doi.org/10.1002/da.1029

World Health Organization (2018). International Classification of Diseases for Mortality and Morbidity Statistics (11th ed.). World Health Organization. https://icd.who.int/browse11/1-m/en

Zerach, G., Greene, T., Ginzburg, K., \& Solomon, Z. (2014). The Relations between Posttraumatic Stress Disorder and Persistent Dissociation among Ex-Prisoners of War: A Longitudinal Study. Psychological Trauma: Theory, Research, Practice, and Policy, 6, 99-108. https://doi.org/10.1037/a0031599 